\section{Hidroneumotórax en paciente inmunocompetente}

\section{Sr. Director:}

Caso clínico: Se trata de una paciente de 33 años diagnosticada de diabetes mellitus tipo 2 en tratamiento con antidiabéticos orales y fumadora activa que consulta por dolor torácico de características pleuríticas, disnea progresiva y fiebre con escalofríos en el último mes habiendo recibido tratamiento antibiótico con claritromicina durante la última semana. En la exploración física presentaba fiebre $\left(\mathrm{T}^{\mathrm{a}} 38^{\circ} \mathrm{C}\right)$ y taquipnea en reposo. Auscultación cardiaca: tonos rítmicos, sin soplos. Auscultación pulmonar: abolición del murmullo vesicular en hemitórax izquierdo con semiología de derrame pleural. Exploraciones complementarias: leucocitos $17350 / \mathrm{mm}^{3}$ (86\% neutrófilos), hemoglobina 10,2 g/dl, Hematocrito 32\%, plaquetas 435.000/ml. Glucosa $304 \mathrm{mg} / \mathrm{dl}$. Gasometría arterial basal: $\mathrm{pH} 7,48, \mathrm{pO}_{2} 61, \mathrm{pCO}_{2} 37, \mathrm{HCO}_{3}-27$, saturación $\mathrm{O}_{2} 93 \%$. Tomografía axial computerizada (TAC) torácica (Fig. 1): derrame pleural con engrosamiento de la superficie pleural con varios niveles hidroaéreos que ocupa el hemitórax izquierdo junto con condensación en lóbulo inferior y superior

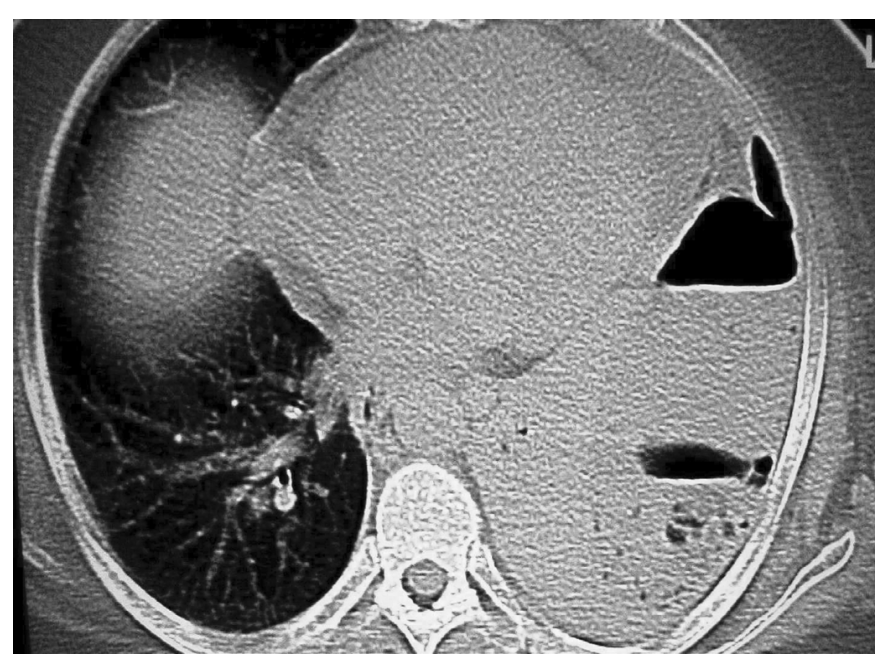

Fig. 1. izquierdos con componente de atelectasia y probable fístula broncopleural.

Se realizó toracocentesis que confirmó la existencia de empiema, colocándose un tubo de drenaje pleural y se administraron 6 dosis de urokinasa. Se tomaron hemocultivos que fueron negativos. En el cultivo del líquido pleural se aislaron Enterococo faecalis y Streptococcus sanguis. En el antibiograma se comprobó la resistencia de Enterococo faecalis a cefalosporinas de tercera generación, quinolonas y clindamicina, siendo sensible a ampicilina. Se inició tratamiento intravenoso durante 2 semanas con ampicilina y posteriormente con amoxicilina-clavulánico durante 3 semanas más tras lo cual la paciente ha permanecido asintomática. Serología VIH: negativa.

Diagnóstico: Neumonía necrotizante y empiema por Enterococo faecalis y Streptococcus sanguis con hidroneumotórax secundario.

Comentario: Se define hidroneumotórax a la coexistencia de aire y líquido en la cavidad pleural, siendo una forma complicada de neumotórax que frecuentemente se asocia a fístula broncopleural. La causa más frecuente es yatrogénica (colocación de vías centrales, toracocentesis). Sin embargo se han descrito casos asociados a neoplasias primarias (mesoteliomas) (1), metastásicas (carcinoma renal) (2) o traumatismos torácicos. Entre las causas infecciosas cabe citar la existencia de hidroneumotórax asociado a empiema piógeno (Klebsiella) (3), Eikenella) (4), tuberculosis (5), nocardia (6) e hidatidosis (7).

El diagnóstico de hidroneumotórax se realiza mediante radiología simple y la TAC, haciendo el diagnóstico diferencial fundamentalmente con el absceso pulmonar. En la radiología simple un punto diferenciador importante es la longitud del nivel hidroaéreo cuando se comparan las proyecciones posteroanterior y lateral. Si la longitud es comparable en ambas proyecciones, probablemente se tratará de un absceso pulmonar. Por el contrario, si la longitud es considerablemente diferente estaremos ante un pioneumotórax (8).

Con la TAC, el nivel hidroaéreo de un hidroneumotórax está en íntima relación con la pared torácica, así el espacio tiene de forma característica un margen regular, liso y netamente definido sin bolsillos laterales y se modifica con los cambios de posición. Por el contrario, el absceso pulmonar es típicamente redondeado, con pared gruesa e irregular siendo el nivel hidroaéreo de igual longitud en todas las posiciones, mientras que la cavidad no varía con los cambios de posición (8). Recientemente se ha demostrado la validez de la ecografía torácica para el diagnóstico 
de hidroneumotórax (9) e incluso para diferenciar los casos asociados a empiema (signo de microburbujas "suspendidas") con una sensibilidad y especificidad del $100 \%$ (10).

\section{F. Jover Díaz, J. Robert Gates, J. M. Cuadrado Pastor}

Unidad de Enfermedades Infecciosas. Servicio de Medicina Interna. Hospital Clínico de San Juan. Alicante

1. Tanaka H, Ivchi K, Nangou S, Ikeda M, Tanaka Y, Mai T. Two cases of recurrent hydropneumothorax caused by malignant pleural mesothelioma. Nippon Kyobu Geka Gakkai Zesshi 1996; 44: 1877-81.

2. Daryanani S, Knausenberger HP, de Takats PG, Guest PJ, Kerr DJ. Spontaneous hydropneumothorax associated with expectoration of a lump of metastatic renal cancer. Clin Oncol (R Coll Radiol) 1997; 9: 262-3.

3. Cook S, Frochaux V. Hydropneumothorax chez un patient souffrant d'une anorexie mentale. Schweiz Med Wochenschr 2000; 130: 1309.

4. Ferrando D, Morales P, Pastor A. Hydropneumothorax in Eikenella corrodens infection. Arch Bronconeumol 1997; 33: 204.

5. Kates DE, Pollack CV. Hydropneumothorax due to tuberculosis. J Emerg Med 1995; 13: 27-30.

6. Fullana Monllor J, Garcia Bermejo PA, Pellicer Ciscar C. Pulmonary abscess and hydropneumothorax secondary to nocardiosis. Arch Bronconeumol 1999; 35: 360-1.

7. Athanassiadi K, Kalavrouziotis G, Loutsidis A, Bellenis I, Exarchos N. Surgical treatment of echinococcosis by a transthoracic approach: a review of 85 cases. Eur J Cardiothorac Surg 1998; 14: 134-40.

8. Light RW, Porcel JM. Derrame pleural paraneumónico y empiema. Med Clin (Barc) 2000; 115: 384-91.

9. Targhetta R, Bourgeois JM, Chavagneux R, Marty-Double C, Balmes P. Ultrasonographic approach to diagnosing hydropneumothorax. Chest 1992; 101: 931-4.

10. Lin FC, Chou CW, Chang SC. Usefulness of the Suspended Microbubble Sign in Differentiating Empyemic and Nonempyemic Hydropneumothorax. J Ultrasound Med 2001;20: 1341-5.

\section{Infección faríngea por Mycoplasma pneumoniae en un adulto joven. Un caso}

\section{Sr. Director:}

Del variado espectro clínico de la enfermedad causada por Mycoplasma pneumoniae (neumonía, otitis, carditis, síndrome neurológico,...) presentamos un caso de infección faringoamigdalar en un varón de 21 años que consultó por un cuadro de una semana de evolución consistente en fiebre alta (hasta $39^{\circ} \mathrm{C}$ ) y molestias en orofaringe. No refería tos.

El paciente aparentaba discreta afectación del estado general, faringe hiperémica con amígdalas agrandadas de forma simétrica con algún ténue exudado que no formaba placas. Existía aumento de volumen del cuello, palpándose pequeñas adenopatías laterocervicales bilaterales a dicho nivel. No había estertores pulmonares.

Entre los datos analíticos destacaba: discreta leucocitosis con fórmula normal, test de anticuerpos heterófilos negativo y las serologías para Borrelia, lúes, Brucella, Coxiella, Rickettsia conorii, Citomegalovirus, EBV, Chlamydia, Legionella, Salmonella, y Toxoplasma no mostraron datos de infección aguda. Sólo fue positiva la serología para Mycoplasma pneumoniae, en base a presencia de anticuerpos Ig M (y ausencia de anticuerpos Ig G) mediante ELISA. Un segundo análisis realizado 8 semanas más tarde mostró aparición de anticuerpos Ig G, con negativización de anticuerpos Ig M, lo cual confirmó el diagnóstico (1). La radiolo- gía torácica en dos proyecciones no había mostrado alteraciones.

Se resolvió la enfermedad tras 10 días de evolución, realizándose tratamiento empírico con amoxicilina-ácido clavulánico 875/125 mg v.o. cada 8 horas, 10 días. Fue diagnosticado de faringoamigdalitis por Mycoplasma pneumoniae.

Mycoplasma pneumoniae ocupa un lugar destacado como causante de neumonía adquirida en la comunidad (2-4) sobre todo en pacientes adolescentes y adultos jóvenes. El diagnóstico se basa en los estudios serológicos dado que el aislamiento del germen es complejo (5).

Aunque clásicamente implicado en infecciones de vías altas como otitis media, síndrome de resfriado persistente (6) y cuadros de tos no productiva (7), su importancia real (cuantitativa) no está bien documentada. Existen pocas referencias en la literatura de Mycoplasma pneumoniae como responsable únicamente de casos de faringoamigdalitis y ello es lo que nos ha animado a comunicar este caso.

\section{J. López Castro, R. Gómez Fernández, J. Marnotes Gonzá- lez, O. Fernández Álvarez}

Servicio de Medicina Interna. Complexo Hospitalario de Ourense

1. Vincent JM, Cherry JD, Nauschuetz WF, Lipton A, Ono CM, Costello $\mathrm{CN}$ et al. Prolonged afebrile nonproductive illnesses in American soldiers in Korea: a serological search for causation. Clin Infect Dis 2000; 30: 534-9.

2. Bosnak M, Dikici B, Bosnak V, Dogru O, Ozkan I, Ceylan A, Haspolat K. Prevalence of Mycoplasma pneumoniae in children in Diyarbakir, the south-east of Turkey. Pediatr Int 2002; 44: 510-2.

3. Hammerschlag MR. Mycoplasma pneumoniae infections. Curr Opin Infect Dis 2001; 14: 181-6.

4. Murie Carrillo de Albornoz M, Hueto Pérez de Heredia J, Sánchez Álvarez J, Tiberio López G, Hernández Palacios H, Rivero Puente A. Epidemiología de las neumonías adquiridas en la comunidad del Área de Salud I de Navarra. Med Clin (Barc) 1991; 97: 50-2.

5. Ferreruela RM, Farga MA, Alcaraz MJ y García de Lomas J. Mycoplasmas aislados de muestras respiratorias. Med Clin 1991; 97: 449-52.

6. Wadowsky RM, Castilla EA, Laus S, Kozy A, Atchison RW, Kingsley LA, Ward JI, Greenberg DP. Evaluation of Chlamydia pneumoniae and Mycoplasma pneumoniae asetiologic agents of persistent cough in adolescents and adults. J Clin Microbiol 2002; 40: 637-40.

7. Sillis M, Harrison B. Clinical aspects of Mycoplasma pneumoniae infection. The Lancet 1992; 339: 301-2.

\section{Toxicidad hepática por flutamida}

\section{Sr. Director:}

La flutamida es un fármaco oral con efecto antiandrogénico utilizado en el tratamiento del cáncer de próstata avanzado (1) Entre sus efectos secundarios se ha descrito que puede producir hepatotoxicidad grave en un porcentaje muy bajo de casos. A continuación presentamos un caso de hepatitis aguda colestásica por flutamida con buena evolución posterior.

Se trata de un paciente de 82 años con antecedentes de hipertensión arterial, hipercolesterolemia e infarto agudo de miocardio 5 años antes, tratado desde hace años con amilorida, hidroclorotiazida, ácido acetilsalicílico, lovastatina y atenolol y que fue diagnosticado en octubre de 2001 de adenocarcinoma de próstata, iniciándose entonces tratamiento con flutamida $(250 \mathrm{mg}$ cada 8 
horas) y leuprorelina (un análogo sintético de la GnRH). A principios de enero de 2002 se objetivó ictericia franca y coluria, por lo que se realizó analítica presentando GPT 1369 U/l, GOT 1102 U/l, bilirrubina 13,6 mg/dl, GGT 586 U/l, LDH 400 U/1, motivo por el que se suspendió la flutamida y se le ingresó en nuestro servicio. El paciente no tenía antecedentes de hepatopatía, abuso de alcohol, transfusiones, drogadicción ni viajes recientes al extranjero.

Clínicamente estuvo prácticamente asintomático durante los tres meses previos, salvo por discreto y progresivo desarrollo de ictericia y coluria, sin presentar fiebre, náuseas, vómitos, prurito, dolor abdominal ni pérdida de peso. En la exploración física sólo cabía reseñar la ictericia.

Durante el ingreso se realizaron las siguientes pruebas: hemograma con 97000 plaquetas y resto normal, tiempo de protrombina $51,5 \%$, tiempo de cefalina 42,4 ", fibrinógeno $250 \mathrm{mg}$, proteínas $5.9 \mathrm{~g} / \mathrm{dl}$ (albúmina 52,1\%, gammaglobulina 27,6\%), resto de bioquímica dentro de los límites normales, serologías de VHA, VHB, VHC y VEB negativas y ecografía abdominal con parénquima hepático sin alteraciones, vía biliar normal y ausencia de ascitis. El paciente permaneció asintomático, fue manejado de forma conservadora y se despigmentó progresivamente, mejorando sus alteraciones analíticas y siendo dado de alta a las 2 semanas con bilirrubina 4,9 mg/dl, GOT $165 \mathrm{U} / \mathrm{l}$, GPT $244 \mathrm{U} / \mathrm{l}$, GGT $415 \mathrm{U} / 1$ y fosfatasa alcalina $130 \mathrm{U} / 1$, con posterior normalización de las mismas, prohibiéndose el tratamiento con flutamida.

La flutamida es un fármaco comercializado desde 1989 que se utiliza como bloqueante hormonal en el cáncer de próstata avanzado (1). Otras veces se ha administrado en la hiperplasia benigna de próstata y en el hirsutismo en mujeres jóvenes, pero su administración en estos casos es muy discutible dado el riesgo de hepatotoxicidad grave $(2,3)$.

Se sabe que puede producir con relativa frecuencia una elevación moderada de las transaminasas (4-6), probablemente sin trascendencia clínica. En otras ocasiones (3) más infrecuentes $(0,5 \%)$, como en este caso, produce una hepatitis aguda que suele manifestarse a las 6-12 semanas del inicio del tratamiento (5), lo que obliga a suspenderlo. Al retirar el fármaco, en la mayoría de los casos el cuadro remite debiendo entonces prohibirse el uso posterior de flutamida. Los casos de fallo hepático fulminante son muy infrecuentes (3) y cursan como una hepatitis aguda grave con rápido deterioro clínico y analítico, produciendo ocasionalmente la muerte o requiriendo trasplante hepático. Se cree que quizás el mecanismo patogénico esté mediado por una reacción inmunogénica (7), aunque no se puede descartar algún papel hepatotóxico directo (4).

Tendremos la sospecha etiológica si existe un período de latencia desde el inicio del tratamiento de 6-12 semanas, tras descartar otras causas de hepatitis aguda y después de observar mejoría analítica al suspender el tratamiento (4). No está justificada éticamente (5) la reintroducción del fármaco para demostrar su papel etiológico y la biopsia no se suele realizar si el cuadro evoluciona favorablemente y la sospecha es alta, ya que no hay datos anatomopatológicos específicos (3).

Por último, es fundamental un control periódico de la función hepática en los primeros 4 a 6 meses del tratamiento en estos pacientes, debiendo siempre advertirles de que consulten en el caso de que aparezca astenia, náuseas, vómitos, ictericia o coluria. Probablemente la polémica se centra en qué hacer en los casos en los que sólo exista una elevación de las transaminasas que no supere 2 ó 3 veces el valor normal, ya que son frecuentes elevaciones discretas transitorias de las enzimas hepáticas durante los 6 primeros meses del tratamiento con normalización posterior sin necesidad de suspender el fármaco $(3,6)$. Quizás en estos casos no sea estrictamente necesario suspender inmediatamente el tratamiento, aunque habrá que valorar la reducción de dosis (5) y se deberán hacer controles analíticos más estrechamente (probablemente semanales), suspendiendo el tratamiento si dichos valores empeoran o si se eleva la bilirrubina. Sin embargo, otros autores abogan por la suspensión del fármaco (4) siempre que se eleven las transaminasas.

Trabajo subvencionado en parte por el Proyecto de Investigación $n^{\circ}$ CM0300045 (Programa de Recursos Humanos y Difusión de la Investigación del Instituto de Salud Carlos III).

\section{Vázquez Romero, L. Gil Grande, P. López Serrano, S. Alemán Villanueva, A. García Plaza}

\section{Servicio de Gastroenterología y Hepatología. Hospital Ramón y Cajal. Madrid}

1. Caballería E, Aragó JV, Sanchís A. Hepatotoxicidad por flutamida. Med Clin (Barc) 1994; 102: 434.

2. Andrade RJ, Lucena MI, Fernández MC, Suárez F, Montero JL, Fraga E, et al. Fulminant liver failure associated with flutamide therapy for hirsutism. Lancet 1999; 353: 983.

3. Fernández Peña CM, Morano Amado LE, Montes Santiago J, Fachal C. Fallo hepático fulminante con evolución fatal inducido por flutamida. Med Clin (Barcelona) 1997; 108: 237-38.

4. Dourakis SP, Alexopoulou AA and Hadziyannis SJ. Fulminant hepatitis after flutamide treatment. J Hepatol 1994; 20 (3): 350-3.

5. Mena FJ, Goyeneche ML, Velicia R, González JM, Bellido J, Caro-Patón A. Hepatitis aguda colestásica secundaria a flutamida. Rev Esp Enferm Dig 1998; 90: 376-7.

6. Rodríguez Gómez SJ, Martínez Moreno J, Martín Arribas MI, Pérez Villoria A, De la Serna Higuera C, Betancourt González A. Fallo hepático fulminante inducido por flutamida. Rev Esp Enferm Dig 2000; 92: 411.

7. Pontiroli L, Sartori M, Pittau S, Morelli S, Boldorini R, Albano E. Flutamide-induced acute hepatitis: investigation on the role of immunoallergic mechanisms. Ital J Gastroenterol Hepatol 1998; 30: 310-4.

\section{Derrame pleural como manifestación del síndrome de hiperestimulación ovárica, tras tratamiento de miomas uterinos con triptorelina}

\section{Sr. Director:}

A raíz de la generalización de técnicas de inducción ovárica en programas de reproducción asistida mediante análogos de las gonadotrofinas (AGT), se describió por primera vez en los años 60 el síndrome de hiperestimulación ovárica (SHO), como una complicación iatrogénica en pacientes tratadas con gonadotrofinas exógenas en el curso de técnicas de fertilización in vitro (1).

El SHO aparece en mujeres jóvenes con antecedentes de reciente inducción ovárica. Su etiología es desconocida, aunque parece estar relacionada con un aumento de la permeabilidad vascular y secuestro de líquidos en tercer espacio, secundario a la liberación de sustancias vasoactivas (2). Clínicamente se presenta como acumulación de líquido en las serosas (peritoneo, pleura y más raramente pericardio) así como hemoconcentración e hipoalbuminemia. Aunque generalmente se resuelve espontáneamente, puede llegar a ser extremadamente grave, produciendo insuficiencia respiratoria o renal, shock hipovolémico, fenómenos embólicos e incluso la muerte (3-4). Presentamos el caso de una paciente que debutó como un derrame pleural de causa desconocida, que había sido tratada previamente con triptorelina (análogo de la GnRH) y que fue finalmente atribuido a este síndrome.

Mujer de 53 años, con antecedente de sangrado y anemia de un año y medio de evolución secundaria a miomas uterinos, que acudió a urgencias de Ginecología por metrorragia severa, apre- 
ciándose una hemoglobina de $6 \mathrm{~g} / \mathrm{dl}$, siendo por ello necesario trasfundir dos unidades de concentrados de hematíes. Asimismo se pautaron 3,75 $\mathrm{mg}$ de Triptorelina (Decapeptyl ${ }^{\circledR}$ ) IM cada 28 días, recibiendo en ese momento la primera dosis. Diecisiete días más tarde acude a urgencias con dolor torácico derecho de dos semanas de evolución, tos seca y fiebre en la última semana, sin otra sintomatología acompañante. A la exploración sólo destacaba una auscultación pulmonar con semiología de derrame pleural derecho. En la analítica existía una ligera leucocitosis de 11.960, con un $12 \%$ de eosinófilos, Hb de 10,6 g/d, VSG de 124, proteínas de $6,5 \mathrm{~g} / \mathrm{dl}$ con albúmina de $3,25 \mathrm{~g} / \mathrm{dl}$, siendo el resto de parámetros del hemograma, bioquímica y coagulación, así como los marcadores tumorales, normales. La radiografía de tórax mostró derrame pleural en 1/3 inferior de hemitórax derecho, sin otras alteraciones. La gasometría arterial (FiO2 de 0.21) presentaba $\mathrm{pH}$ de $7,50, \mathrm{pO}_{2}$ de $65, \mathrm{pCO}_{2}$ de $34,1, \mathrm{HCO}_{3}$ de 26 y saturación de $94 \%$. Se realizó toracocentesis diagnóstica obteniendo líquido de aspecto serohemático con características de exudado, con 9.300 leucocitos (59\% neutrófilos y $41 \%$ linfocitos), 38.300 hematíes, $\mathrm{pH}$ 7,40, glucosa de $90 \mathrm{mg} / \mathrm{dl}$, LDH 756 U/L, proteínas 5,1 g/dl, colesterol $157 \mathrm{mg} / \mathrm{dl}$, ADA $28 \mathrm{U} / \mathrm{L}$ y CEA 1,6 U/L. La citología y microbiología del líquido fueron negativas y en las muestras de biopsia pleural no se apreciaron granulomas ni hallazgos sugestivos de malignidad. Se realizó broncoscopia que fue normal, con negatividad en las muestras de broncoaspirado, tanto para microbiología como para citología. La gammagrafía de ventilación/perfusión mostró baja probabilidad para TEP y un ecodoppler de MMII fue normal. En la ecografía abdominal había leves signos de ascitis y se realizó una mamografía que fue negativa. Ante la inexistencia de hallazgos de patología neoplásica, infecciosa o tromboembólica y con el antecedente farmacológico ya señalado, la paciente fue diagnosticada de SHO y tratada con Indometacina $50 \mathrm{mg} / 8 \mathrm{~h}$, obteniendo como resultado la rápida resolución del derrame en quince días sin precisar drenaje del mismo, desapareciendo la sintomatología.

El derrame pleural como manifestación del SHO aparece en el $32 \%$ de las formas graves del mismo (1). Puede presentarse tanto en forma de trasudado como de exudado, y generalmente aparece en el lado derecho, debido al menor drenaje linfático en esta zona (2). Es infrecuente su presentación de forma aislada, siendo más común que acompañe a otras manifestaciones severas como la ascitis, hemoconcentración y oliguria. El diagnóstico se realiza por exclusión y el tratamiento es sintomático, no siendo necesaria generalmente la toracocentesis terapéutica, que se reserva para derrames rápidamente progresivos o para casos de insuficiencia respiratoria (4).

En general, el síndrome se puede clasificar en leve, moderado, severo y crítico, en función de los hallazgos clínicos y analíticos. La aparición de derrame pleural lo coloca ya dentro de las formas graves. Suele comenzar entre los 7 y 14 días tras la estimulación, estando relacionada su severidad con el momento de aparición, de manera que cuando se manifiesta antes de los 7 días suele tratarse de formas más leves de la enfermedad. Se consideran factores de riesgo ser menor de 35 años, un número de folículos mayor de 10, una concentración de estradiol en plasma mayor de 2.000 $\mathrm{pg} / \mathrm{ml}$, el ovario poliquístico y el embarazo, puesto que las gonadotrofinas endógenas pueden estimular y prolongar el cuadro (3).

Los casos descritos en la literatura son escasos, y la mayoría de ellos aparecen en el contexto de la estimulación ovárica, como parte de las técnicas de inducción del embarazo (5). En el caso de nuestra paciente, el desencadenante fue el tratamiento con análogos de las gonadotrofinas como tratamiento de una patología ginecológica, en este caso el mioma uterino. Probablemente tanto el desarrollo de las técnicas de reproducción como el uso de análogos en patologías que requieren la supresión del eje hipofisario, como son la pubertad precoz, los miomas uterinos y la endometriosis, entre otros, hagan que nos encontremos el SHO con mayor frecuencia, obligando al clínico a tenerlo presente como diagnóstico diferencial en caso de aparición de derrame pleural en pacientes sometidos a tratamientos de este tipo, así como a estar atentos para detectar la enfermedad en su inicio, evitando así su progresión a formas más graves.

\section{A. L. Andreu, E. Chiner, E. Gómez Merino, J. Signes-Costa}

Sección de Neumología. Hospital Universitario San Juan de Alicante

1. Laguno M, Queralt C, Casademont J. Right pleural efusión as an isolated manifestation of ovarian hyperstimulation síndrome. Med Clin (Barc) 2000; 115: 438.

2. Man A, Schwarz Y, Greif J. Pleural effusion as a presenting symptom of ovarian hyperstimulation syndrome. Eur Respir J 1997; 10: 2425-6.

3. Roden S, Juvin K, Homasson JP, Israel-Biet D. An uncommon etiology of isolated pleural effusion. the ovarian hyperstimulation syndrome. Chest 2000; 118: 256-8.

4. Moggi V, Nicolau F, Romero A, Ramis P. El síndrome de hiperestimulación ovárica como causa de derrame pleural. Arch Bronconeumol 1999; 35: 192-3.

5. Abramov Y, Elchalal U, Schenker JG. Pulmonary manifestations of severe ovarian hyperstimulation syndrome: a multicenter study. Fertil Steril 1999; 71: 645-51.

\section{Prevalencia de síndrome metabólico en pacientes con diabetes tipo 2}

\section{Sr. Director:}

La diabetes mellitus hace que desaparezca el efecto protector del sexo femenino sobre la aparición de cardiopatía isquémica, como fue puesto de manifiesto en las cohortes de Framingham (1) y de Rancho Bernardo (2); en este último estudio el riesgo relativo de cardiopatía isquémica en diabéticos versus no diabéticos fue 1,9 para varones y 3,3 para mujeres. Por otro lado el síndrome metabólico, una asociación de alteración del metabolismo de la glucosa, obesidad abdominal, dislipemia e hipertensión, se ha asociado a la aparición de diabetes mellitus y de enfermedad cardiovascular (3). Una explicación posible para el efecto más adverso de la diabetes sobre la mujer puede ser la presencia de un perfil de factores de riesgo más aterogénico en mujeres diabéticas versus no diabéticas que entre varones diabéticos versus no diabéticos (4). El objetivo de nuestro estudio fue evaluar si había una mayor prevalencia de componentes del síndrome metabólico en mujeres que en varones con diabetes tipo 2.

En este estudio descriptivo transversal incluimos pacientes con diabetes tipo 2 atendidos en consultas externas de un hospital comarcal. Se excluyeron pacientes con proteinuria y/o creatinina $>3 \mathrm{mg} / \mathrm{dl}$. Se determinó la prevalencia de componentes del síndrome metabólico como definidos por la OMS (5): hipertensión (presión sistólica > $160 \mathrm{mmHg}$ y/o presión diastólica > $90 \mathrm{mmHg}$ y/ó tratamiento con hipotensores), dislipemia (triglicéridos $>150$ $\mathrm{mg} / \mathrm{dl}$ y/o HDL $<35 \mathrm{mg} / \mathrm{dl}$ en el varón o menor de $40 \mathrm{mg} / \mathrm{dl}$ en la mujer), obesidad (índice de masa corporal (peso en $\mathrm{kg} / \mathrm{talla}$ al cuadrado en metros) $>30$ y/o cociente cintura cadera $>0,9$ en el varón o mayor de 0,85 en la mujer) y microalbuminuria (tasa de excreción de albúmina $>20 \mathrm{mcg} /$ minuto). Una persona con diabetes tiene el síndrome metabólico si cumple 2 o más de los criterios anteriores. 
TABLA I

CARACTERÍSTICAS GENERALES Y PREVALENCIA DE COMPONENTES DEL SÍNDROME METABÓLICO EN EL GRUPO TOTAL Y CLASIFICADO POR SEXOS

\begin{tabular}{|c|c|c|c|c|}
\hline Variables & Total & Mujeres & Varones & $p$ \\
\hline Número de pacientes & 318 & 188 & 130 & \\
\hline Edad (años) & $64,6(9,1)$ & $65,1(8,9)$ & $63,7(9,3)$ & ns \\
\hline Evolución (años) & $10,8(7,7)$ & $11,5(7,9)$ & $9,8(7,3)$ & ns \\
\hline $\mathrm{CC}$ & $0,98(0,096)$ & $0,97(0,12)$ & $0,99(0,04)$ & 0,025 \\
\hline TAS (mmHg) & $143(19,5)$ & 147 (19) & 138 (19) & $<0,0001$ \\
\hline TAD (mmHg) & $79,3(11,7)$ & $80,5(11,6)$ & $77,5(11,8)$ & 0,021 \\
\hline $\mathrm{HDL}(\mathrm{mg} / \mathrm{dl})$ & $44(11,9)$ & $45(11,7)$ & $42(11,9)$ & 0,018 \\
\hline $\mathrm{LDL}(\mathrm{mg} / \mathrm{dl})$ & $149(33,4)$ & $148(32,5)$ & $151(34,7)$ & ns \\
\hline Prevalencia obesidad (\%) & 97,2 & 96,3 & 98,5 & ns \\
\hline Prevalencia HTA (\%) & 55 & 65,4 & 40 & $<0,0001$ \\
\hline Prevalencia dislipemia (\%) & 41,8 & 46,3 & 35,4 & 0,05 \\
\hline Prevalencia MAL (\%) & 23,9 & 21,8 & 26,9 & ns \\
\hline
\end{tabular}

$\mathrm{IMC}=$ índice de masa corporal; $\mathrm{CC}=$ cociente cintura cadera; TAS= presión arterial sistólica; TAD= presión arterial diastólica; HTA= hipertensión arterial; $M A L=$ microalbuminuria

Las variables cuantitativas se describen con su media y desviación estándar (DE) y las cualitativas con distribución de frecuencias. La comparación de variables cuantitativas se realiza con t de Student para grupos independientes y la de variables cualitativas con chi cuadrado. Se consideran significativas las asociaciones con $\mathrm{p}<0,05$.

Se incluyeron 318 pacientes con una edad de 64.6 años (DE $9,1)$ y un tiempo de evolución de la diabetes de 10,8 años (DE $7,7)$. Hubo 188 mujeres $(59,1 \%)$ y 130 varones $(40,9 \%)$. La distribución de factores de riesgo pertenecientes al síndrome metabólico para el grupo total y por sexos quedan reflejados en la tabla 1. Aunque la prevalencia de síndrome metabólico fue similar en ambos sexos (79,8\% mujeres vs $73,1 \%$ varones; $\mathrm{p}=\mathrm{ns})$, las mujeres tenían mayores prevalencias de hipertensión y de dislipemia, por lo que el número medio de componentes del síndrome metabólico fue superior en mujeres (2,3 en mujeres vs 2,01 en varones; $\mathrm{p}=0,003)$.

Los principales hallazgos de este trabajo han sido dobles. Por un lado la detección de una elevada prevalencia de componentes del síndrome metabólico en nuestra muestra de diabéticos tipo 2. Por otro lado la constatación de mayor prevalencia de hipertensión y dislipemia en mujeres que en varones diabéticos.

El síndrome metabólico, también llamado síndrome de resistencia insulínica, incrementa el riesgo cardiovascular en sujetos sin diabetes mellitus (6). También se ha descrito que la resistencia insulínica, determinada con modelo HOMA, es un predictor independiente de enfermedad cardiovascular en pacientes con diabetes tipo 2 (7). En nuestro trabajo la prevalencia de síndrome metabólico fue elevada, aunque similar a la descrita en la cohorte de Botnia para pacientes con diabetes tipo 2 con una definición similar: $78 \%$ mujeres y $84 \%$ varones (8).

En nuestro estudio constatamos mayores prevalencias de dislipemia e hipertensión en mujeres que en varones diabéticos. Por debajo de los 55 años de edad la incidencia de cardiopatía isquémica es en el varón 3 veces superior respecto a la mujer; no obstante dicha diferencia se anula en pacientes con diabetes tipo 2 . En el Strong Heart Study (4) se midieron factores de riesgo cardiovascular en 1.846 hombres y 2.703 mujeres de 45 a 74 años. En análisis de covarianza hubo un termino de interacción significativo de sexo por diabetes para cociente cintura cadera, LDLcolesterol, HDL-colesterol, apoB, apoA1, tamaño de LDL y fibrinógeno, lo que confirmaba un efecto mas deletéreo de la dia- betes en la mujer, en parte mediado por estas variables intermedias. En otros estudios (1) también se ha comunicado mayor prevalencia de hipertensión en mujeres diabéticas que en hombres diabéticos.

Una limitación de nuestro trabajo fue haber seleccionado una muestra de pacientes atendidos en consulta especializada, por lo que los resultados podrían no ser generalizables a la población total de pacientes diabéticos. No obstante, dada la elevada prevalencia detectada en nuestro estudio, consideramos prioritario investigar la presencia de factores de riesgo de enfermedad cardiovascular en pacientes con diabetes tipo 2 . No hay que olvidar que la enfermedad cardiovascular constituye la principal causa de mortalidad en el paciente diabético (9) y que recientemente ha quedado demostrada la eficacia a largo plazo de una intervención multifactorial (10).

Podemos concluir diciendo que en nuestro medio los pacientes con diabetes tipo 2 tienen una elevada prevalencia de componentes del síndrome metabólico, especialmente las mujeres, lo que podría explicar el efecto más deletéreo de la diabetes tipo 2 sobre el sexo femenino.

\section{J. A. Gimeno Orna, B. Boned Juliani ${ }^{1}$, L. M. Lou Arnal}

Sección de Medicina Interna. ${ }^{1}$ Sección de Bioquímica. Hospital Comarcal de Alcañiz. Teruel

1. Kannel WB, McGee DL: Diabetes and cardiovascular disease: the Framingham Study. JAMA 1979; 241: 2035-2038.

2. Barrett Connor EL, Cohn BA, Wingard DL, Edelstein SL. Why is diabetes mellitus a stronger risk factor for fatal ischemic heart disease in women than in men?. The Rancho Bernardo Study. JAMA 1991; 265: 627-631.

3. Liese AD, Mayer-Davis EJ, Haffner SM. Development of the multiple metabolic syndrome: an epidemiologic perspective. Epidemiol Rev 1998; 20: 157-172.

4. Howard BW, Cowan LD, Go O, Welty TK, Robbins DC, Lee ET. Adverse effects of diabetes on multiple cardiovascular disease risk factors in women. The Strong Heart Study. Diabetes Care 1998; 21: 12581265

5. Alberti KGMM, Zimmet PZ, for the WHO Consultation: Definition, diagnosis and classification of diabetes mellitus and its complications. Part 1: Diagnosis and classification of diabetes mellitus, provisional 
report of a WHO consultation. Diabet Med 1998; 15: 539-553.

6. Lakka HM, Laaksonen DE, Lakka TA et al. The metabolic syndrome and total and cardiovascular disease mortality in middle-aged men. JAMA 2002; 288: 2709-2716.

7. Bonora E, Formentini G, Calcaterra F et al. HOMA-estimated insulin resistance is an independent predictor of cardiovascular disease in type 2 diabetic subjects. Diabetes Care 2002; 25: 1135-1141.

8. Isomaa $\mathrm{B}$, Almgren $\mathrm{P}$, Tuomi $\mathrm{T}$ et al. Cardiovascular morbidity and mortality associated with the metabolic syndrome. Diabetes Care 2001; 24: 683-689.

9. Stamler J, Vaccaro O, Neaton JD, Wentworth D for the Multiple Risk Factor Intervention Trial Research Group: Diabetes, other risk factors, and 12-yr cardiovascular mortality for men screened in the Multiple Risk Factor Intervention Trial. Diabetes Care 1993; 16: 434-444.

10. Gaede P, Vedel P, Larsen N. Multifactorial intervention and cardiovascular disease in patients with type 2 diabetes. N Engl J Med 2003; 348: 383-393.

\section{Patrón infiltrativo hepático y dolores óseos}

\section{Sr. Director:}

El thorotrast (Th) es una suspensión coloidal al 25\% de dióxido de torio, que se utilizaba en la práctica clínica como medio de contraste (1). Mc Mahon publica el primer caso de neoplasia inducidad por Th (2). A principios de los 50 deja de emplearse calculándose entre 50.000 y 100.000 personas a los que se les administró dicho producto (3). Aportamos un nuevo caso clínico de colangiocarcinoma inducido por Th.

Varón de 73 años, con antecedentes de realización de estudio angiográfico con dióxido de torio hace más de 50 años, para diagnóstico de hemorragia subaracnoidea, que ingresa por patrón infiltrativo hepático, síndrome general y dolores óseos de tres meses de evolución. Además dolor en hipocondrio derecho sin relación con la ingesta e intensos dolores en raquis dorsal, que no ceden con analgesia habitual. A la exploración: dolor a la palpación en hipocondrio derecho y a la presión selectiva de espinosas en región dorsal y lumbar, no soplos abdominales.

Datos Complementarios: AST: $42 \mathrm{UI} / \mathrm{L}, \mathrm{ALT}$ : $51 \mathrm{UI} / \mathrm{L}$, fosfatasa alcalina: 347 U/L, GGT: 369 U/L. Rx de tórax: pérdida de altura del espacio discal D11-D12 y D12-L1 y restos de contraste linfográfico. Tac Abdominal: Lesión focal hepática hipodensa de $5 \mathrm{cn}$ a nivel del segmento 1 adyacente a la desembocadura de las suprahepáticas, destacando acúmulos de densidad metálica hepatoesplénicas, además se visualiza otra lesión de $3 \mathrm{~cm}$ en el segmento VIII subdiafragmática. PAAF hepática ecodirigida: grumos de células neoplásicas con características de malignidad de apariencia epitelial y material intracitoplasmático de tipo biliar, sugestivo de tumor maligno epitelial hepático primario.

A los 10 días del ingreso síndrome confusional agudo y cuadro comicial generalizado, siendo demostradas múltiples metástasis cerebrales, siendo exitus por neumonía por aspiración, no se concedió la necropsia.

El Th posee una vida media biológica de 400 años, dando lugar en su desintegración a derivados radiactivos que emiten radiaciones alfa $(90 \%)$, beta $(9 \%)$ y gamma (1\%), siendo las alfa las más nocivas para el organismo (4). Tras su administración es captado por el sistema monunuclear fagocítico para depositarse en hígado, bazo, ganglios linfáticos, médula ósea y en menor proporción por el resto de los órganos $(1,5)$. El tiempo de latencia desde la administración del contraste y la aparición de tumores oscila entre 3 y 35 años (15), habiendo sido descrito como mayor latencia el caso de un paciente que a los 60 años de la exposición desarrolló angiosarcoma y colangiocarcinoma hepáticos simultáneamente (5). Ya que el Th fue utilizado hasta 1955, y dado el tiempo de latencia estimado has-

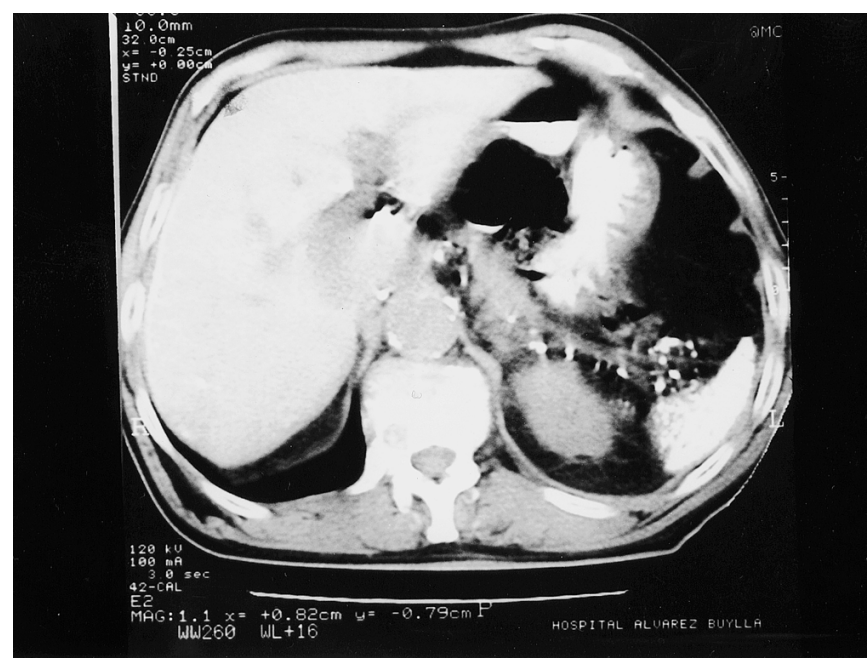

Fig. 1. Lesión hipodensa focal hepática de $5 \mathrm{~cm}$ a nivel de segmento I, adyacente a la desembocadura de las suprahepáticas, y otra lesión de $3 \mathrm{~cm}$ en segmento VIII subdiafragmático y acúmulos con densidad metálica adyacentes a bazo.

ta la aparición de tumores hepáticos, se supone que tumores hepáticos inducidos por Th, pueden ser diagnosticados hasta el año 2015 (6). Las neoplasias relacionadas con el Th se observan en cualquier órgano, pero principalmente son consecuencia de afectación del sistema hepatobiliar, bazo y médula ósea (6), siendo el colangiocarcinoma el más frecuente (43\%), angiosarcoma (38\%), hepatocarcinoma $(16 \%)$, tumores mixtos $(3 \%)(1)$. La patología derivada de la afectación de médula ósea se traduce en leucemia mieloide aguda, monocítica aguda, mieloide crónica, aplasia medular y mielofibrosis (5). Es característica la agresividad clínica tras el diagnóstico de neoplasia hepática inducida por $\mathrm{Th}$, siendo la supervivencia de escasos meses (1-5), sin existir un tratamiento efectivo. Se asume una relación inversa entre la dosis administrada y la aparición de los tumores, aunque no es un parámetro válido para aventurar un diagnóstico de presunción (5). Desde el punto de vista radiológico el diagnóstico es relativamente sencillo al observar acúmulos con densidad metálica a nivel de hígado, bazo o zonas adyacentes, que hace sospechar la naturaleza del depósito (1), además la utilización del TAC puede determinar las concentraciones de torio en hígado y bazo $(1,6)$.

Diagnóstico: colangiocarcinoma inducido por thorotrast.

\section{R. Gómez de la Torre, F. Cadenas ${ }^{1}$, A. Milla Crespo, J. Fer- nández Bustamante}

\section{Medicina Interna. ${ }^{\prime}$ Digestivo-Unidad de Endoscopias. Hospital Álvarez-Buylla. Mieres, Asturias. SESPA}

1. Martí Vicente. A, González Carro. P, Enríquez J, Vilardell F. Thorotrastosis hepática. Rev Esp Enf Digest 1993; 83, 1: 16-19.

2. Mac Mahon HE, Murphy AS, Bates MI. Endothelial-cell sarcoma of the liver following Thorotrast injections. Am J Pathol 1947; 23: 585-611.

3. Selinger M, Koff RS. Thorotrast and the liver: a reminder. Gastroenterology 1975; 68: 799-803.

4. Iwamoto KS, Mizuno T, Kurata A, Masuzawa M, Mori T, Seyama T. Múltiple, unique, and common p53 mutations in a thorotrast recipient with four primary cancers. Hum Pathol 1998; 29 (4): 412-6.

5. Lee FI, Tharakan J, Vasudev K, E. T. Isaacs, P. Malignant hepatic tumours associated with previous exposure to Throtrast: four cases. Eur J Gastroenterol Hepatol 1996; 8: 1121-1124.

6. Jansen TLTA, Meijer JWR, Kesseiring OHW, Mulder CJJ: Synchronous hepatic tumours 60 year after diagnostic Thorotrast use. Eur J Gastroenterol Hepatol 1992; 4: 753-755. 


\section{Esofagitis herpética asociada a esofagitis por Candida albicans en paciente inmunocompetente}

\section{Sr. Director:}

La esofagitis por virus herpes $(\mathrm{EH})$ se presenta en pacientes con depresión del sistema inmune (SIDA, trasplantados y neoplasias especialmente hematológicas), siendo su aparición en pacientes inmunocompetentes muy poco común, especialmente si está asociada a una esofagitis por candida (EC) de la que únicamente hemos encontrado un caso descrito previamente (1-3) por lo cual creemos interesante el aportarla.

Mujer de 74 años, antecedentes de diabetes mellitus. Refiere tos, con expectoración amarillenta, disnea de reposo de 4 días de evolución. Exploración física. $\mathrm{T}^{\mathrm{o}} 36^{\circ} \mathrm{C}$. TA 180/70. Frecuencia respiratoria $24 \mathrm{rpm}$. Frecuencia cardiaca $85 \mathrm{ppm}$. Paciente cosnciente, colaboradora, disneica. Auscultación pulmonar; hipoventilación global, crepitantes basales bilaterales. Resto exploración normal.

Analítica. Gasometría: $\mathrm{PO}_{2} 66, \mathrm{PCO}_{2} 35$. Hemograma normal, VSG $35 \mathrm{~mm}$, glucosa $150 \mathrm{mgr} / \mathrm{dl}$, resto analítica sin datos destacables. Serologías: HIV negativo, virus herpes simple. Ig G positiva, IgM negativa, , varicella-zoster negativa, citomegalovirus negativa.

La paciente fue tratada con levofloxacino $400 \mathrm{mg}$ /día vía oral durante 5 dias, aerosolterapia con salbutamol y bromuro de ipratropion, prednisona, oral $40 \mathrm{mg} /$ día y omeprazol, oral 20 $\mathrm{mg}$ /día.La evolución fue favorable con rápida mejoría clínica, al $5^{\circ}$ día presenta bruscamente dolor retroesternal y odinofagia. Grastroscopia. Esófago recubierto en su totalidad por una capa blanquecina que sangra al roce, se realizan cepillados y biopsias. Anatomía patológica. Presencia células escamosas con alteraciones reactivas, abundantes esporas y pseudohifas de Albicans, así mismo se observan abundantes células escamosas sueltas o en placas planas, con núcleos agrandados y amoldados de cromatina esmerilada y multinucleación, compatible con infección herpética y esofagitis por Candida.

La paciente fue tratada con fluconazol $100 \mathrm{mg} /$ día durante 14 días y retirada progresiva de esteroides, estando a las 72 horas asintomática. La gastroscopia de control a los 15 días fue normal.

$\mathrm{La} \mathrm{EH}$ en el paciente inmunocompetente es una entidad infrecuente. Puede presentarse como infección primaria o reactivación, en nuestro caso creemos que está asociada a esta última $(1,4)$. La EH puede aparecer como complicación de la corticoterapia oral o parenteral, hay datos que sugieren que la asociación de esteroides, con omeprazol y antibioterapia serían factores que favorecerían el desarrollo de EC $(3,5)$. Existen antecedentes de patología respiratoria previa entre 3-21 días antes de la EH en un $23,7 \%$. El inicio es agudo $(83,9 \%)$ y se caracteriza por odinofagia, dolor retroesternal y fiebre, dichas manifestaciones son similares a las de la esofagitis por $(1,4)$. La esofagocopia, tanto en la EH como EC muestra una mucosa inflamada y friable con cambios hemorrágicos y exudados (6). La presencia de Albicans en el cepillado es del 92-100\% sugiriendo el diagnóstico de esofagitis siempre que el hongo aparezca en forma de hifas y/o mezclado con células epiteliales viables (1). En la EH la citología ea altamente específica y sensible $(90,9 \%)$ y sugiere etiología viral en el $100 \%$, mientras que en los cultivos virales la positividad es hasta el $95,8 \%$. La serología habitualmente tiene un valor limitado ya que un $90 \%$ de la población sana puede haber tenido exposición previa al virus herpes y tener la IgG positiva con o sin anticuerpos IgM. Sin embargo la seroconversión puede ser diagnostica $(4,7)$. El tratamiento de la EH es con aciclovir oral o intravenoso, sin embargo en pacientes inmunocompetentes no es preciso al ser un proceso autolimitado. Y no existir ensayos clínicos controlados del efecto del tratamiento con aciclovir en adultos sanos
$(8,9)$. La EC el tratamiento está condicionado a la severidad de la infección pudiéndose tratar con antifúngicos no absorbibles como clotrimazol o la nistatina, en casos severos fluconazol o ketoconazol (1), en nuestro caso se decidió tratamiento con fluconazol 100 mgr día durante 14 días.

\section{J. Marti Cabané, M. Álvarez Rubio}

\section{Hospital de Zumárraga. Guipúzcoa}

1. Arenas JL. Montalvo I I. Gil I. Esofagitis infecciosas. Gastroenterol Hepatol 1999; 22: 302-308.

2. Yacono JV. Type I herpes simplex esophagitis with candidal esophagitis in an immunocompetent host. N Y S J Med 1985; 85 (11): 656-658.

3. Antón E. Álvarez M. Esofagitis herpética ulcerativa en paciente inmunocompetente. Rev Esp Enferm Dig (Madrid) 2001; 93: 818-819.

4. Ramanathan J, Rammouni M, Baran Jr J, Khatib R. Herpes simplex virus esophagitis in the immnucompetent host:an overview. Am J Gastroenterol 2000; 95: 2171-2176.

5. Chocarro A. Galindo F. Ruiz-Irastorza G, et al. Risk factors for esophageal candidiasis. Eur J Clin Microbiol Infectious Dis 2000: 19; 96-100.

6. Kirsch M. Herpes esophagitis in an immunocompetent host.Am Fam Phys 1998; 57: 1780-81.

7. Varsky CG. Yahni VD. Freire MC, et al. Esophageal pathology in patients with the AIDS virus.Etiology and diagnosis(Abstract). Acta Gastroenterol Latinoam 1991; 21: 67-83.

8. Pamuk OM, Pamuk GE, Celik AF, Ortrk R, Aktuglu Y. Herpes simplex virus esophagitis in an immunocompetent host with sepsis. Am J Gastroenterol 2001; 96: 2264-2266.

9. Zagnoon A, Kulkarni P. Herpes simplex esophagitis. Am J Gastroentrol 1999; 94: 1708-1709.

\section{Experiencia de carcinoma de tiroides en un hospital comarcal: estudio de 21 casos}

\section{Sr. Director:}

El cáncer de tiroides es un tumor infrecuente, representa el $1 \%$ de todas las neoplasias. Sin embargo, es el tumor endocrino maligno más frecuente. El $90 \%$ son tumores bien diferenciados, siendo los más frecuentes el carcinoma papilar, el folicular y el de células de Hürthle. El resto, corresponden a carcinomas medulares, anaplásicos o linfomas (1).

Se han estudiado 21 pacientes diagnosticados en el servicio de medicina interna del hospital de Zumárraga en los últimos 9 años. De los 21 pacientes, 14 son mujeres y 7 varones, con edades comprendidas entre 17-71 años (42,7 $\pm 16,5$ años). El motivo de la consulta fue la aparición de una tumoración en región cervical (75\%) de 4,2 \pm 4 meses de evolución. En el $95 \%$ de los pacientes existía bocio, siendo uninodular en el $60 \%$ y multinodulares en el $35 \%$. Las adenopatías fueron palpables en 7 pacientes y en una paciente fue el primer signo de la enfermedad. 18 estaban eutiroideos y 3 presentaban hipertiroidismo. La gammagrafía tiroidea mostró la presencia de un nódulo frío en el 53\%. Se realizó PAAF a 19 pacientes, y fue positiva en 15 . La tiroglobulina previa a la intervención se determinó en el $48 \%$, encontrándose elevada en el $91 \%$. El diagnóstico se estableció previo a la intervención en el $65 \%$. Todos los pacientes fueron sometidos a tiroidectomía total y en 4 se asoció un vaciado cervical funcional unilateral y en 1 fue bilateral. La lesión fue unilateral en $78 \%$ con un tamaño de $1,9 \pm 1,2 \mathrm{~cm}$ y en un $22 \%$ se observó afectación del lóbulo contralateral. La correlación entre la PAAF previa y el 
diagnóstico final fue del 79\%. Histológicamente los tumores fueron: 15 carcinomas papilares (NOS 11, variante folicular 2, células altas 2), 4 carcinomas foliculares, y 2 carcinomas de células de Hürtle.

A todos los pacientes se les realizó rastreo corporal, tratamiento ablativo de restos con I 131 y tratamiento con T4 a dosis supresoras. La tiroglobulina se determinó en el $82 \%$ de los pacientes en los controles evolutivos siendo normal en todos excepto en 1 paciente. La recidiva tumoral se objetivó en 2 pacientes ( 1 carcinoma papilar y 1 carcinoma de células de Hürthle). La duración del seguimiento ha oscilado entre 1 y 8 años $(4,65 \pm 2,6$ años). Un paciente con carcinoma papilar falleció a los 18 meses del diagnóstico. En tres pacientes ( 2 c. papilar y 1 carcinoma de células de Hürthle) se perdió el seguimiento. Actualmente todos los pacientes controlados se encuentran asintomáticos sin observar recidivas.

La manifestación clínica más característica es la aparición de un nódulo solitario de tamaño y duración variables, aunque en ocasiones la presencia de adenopatías cervicales puede ser el primer hallazgo $(2,3)$. Los estudios isotópicos al igual que la ecografía son inespecíficos y muestran la presencia de un nódulo tiroideo frío, con un patrón ecográfico sólido, quístico o mixto (4). La punción aspiración con aguja fina (PAAF) es la base en el diagnóstico de lesiones tiroideas. En manos expertas se puede llegar a un diagnóstico en el $90 \%$ de los casos. Sin embargo, en tumores foliculares y de células de Hürthle, las características citológicas son insuficientes, ya que la malignidad se basa principalmente en la presencia de invasión capsular, hecho imposible de valorar en una citología. En estos casos el diagnóstico por PAAF es del 10$20 \%(5)$.

El tratamiento se basa en tiroidectomía total, asociado a tratamiento con radioyodo de los restos tiroideos y tratamiento con T4 a dosis supresoras. La lobectomía aislada puede presentar entre un 5-10\% de recurrencia en el lóbulo contralateral. Unicamente estaría indicada en el microcarcinoma papilar $(<1$ $\mathrm{cm}$ ), descubierto con posterioridad a la cirugía tiroidea benigna, o al carcinoma folicular $(<4 \mathrm{~cm})$ mínimamente invasivo sin invasión vascular, no identificado como maligno en la PAAF (6).

El seguimiento se realiza mediante determinación de niveles de tiroglobulina con supresión de hormona tiroidea o mediante estimulación con rhTSH. Para detectar recidiva de la enfermedad se realizan rastreos isotópicos. En los pacientes a los que se les ha realizado únicamente lobectomía, se realiza ecografía cervical $(5,6)$.

\section{Valentí Ponsa, J. Martí Cabané ${ }^{1}$}

Servicios de Anatomía Patológica y ${ }^{1}$ Medicina Interna. Hospital de Zumárraga. Guipúzcoa

1. Hundahl SA, Cady B, Cuningham MP, et al. Initial results from a prospective cohort study of 5583 cases of thyroid carcinoma treated in the United States during 1996. Cancer 2000; 89: 202-17.

2. Mazzaferri EL, Jhiang SM. Long term impact of inicial surgical and medical therapy on papillary and follicular thyroid cancer. Am J Med 1994; 97: 418-28.

3. Clark OM, Duh GS. Thyroid cancer. Med Clin North A 1991; 75: 21134.

4. Escribano JL, Jimeno JM. Cancer de tiroides. Medicine 2000; 8: 910-8.

5. Singer PA, Cooper DS, Daniels GM, et al. Treatment guidelines for patiens with thyroid nodule and well differentiated thyroid cancer. Arch Intern Med 1996; 156: 2165-72.

6. Mazzaferri EL, Kloos RT. Current approaches to primary therapy for papillary and follicular thyroid cancer. J Clin Endocrinol Metab 2001; 86: 1447-63.
¿Existe permeabilidad completa de la vena previamente trombosada cuando finaliza el tratamiento anticoagulante en la trombosis venosa profunda idiopática?

\section{Sr. Director:}

La ultrasonografía de compresión venosa o ecodoppler de miembros inferiores constituye la prueba fundamental para el diagnostico de la trombosis venosa profunda, teniendo una sensibilidad y especificidad por encima del $96 \%$ (1). Además permite realizar el diagnóstico diferencial con otras causas, como quiste de Baker, hematomas, tumores, etc (1). La sensibilidad de ésta prueba disminuye cuando la trombosis venosa profunda afecta a venas distales (2). La determinación del dímero D también resulta útil para el diagnóstico de exclusión de la trombosis venosa profunda, así ante niveles elevados hay que pensar en una mayor extensión (3), pero se pueden encontrar falsos negativos en la trombosis venosa profunda de localización distal y después de dos semanas de haberse iniciado el tratamiento anticoagulante (4). La administración relativamente cómoda de las heparinas de bajo peso molecular, sin necesidad de efectuar controles analíticos han permitido el tratamiento ambulatorio de ésta enfermedad en múltiples ocasiones (5). Actualmente se considera que la duración del tratamiento anticoagulante en pacientes diagnosticados de trombosis venosa profunda idiopática debe ser de unos 6 meses (6). Recientemente hemos realizado un estudio en que analizábamos el tiempo necesario para conseguir la permeabilidad completa de la vena previamente ocluida, mientras recibía tratamiento anticoagulante, para valorar si el tiempo aceptado como necesario, habitualmente de unos 6 meses, resultaba suficiente para que se obtuviera la permeabilidad completa de la vena trombosada.

Los criterios diagnósticos se habían realizado mediante la conjunción de los datos clínicos, elevación del dimero D y visualización de la oclusión completa en la ultrasonografía de compresión venosa, Incluimos 13 enfermos diagnosticados de trombosis venosa profunda idiopática, 12 varones y 1 mujer, con una edad media de 70 años.

Presentaban trombosis venosa profunda con afectación íliofemoral 6 casos (46\%) y femoropoplítea 7 casos $(54 \%)$.

Se efectúo tratamiento con heparina de bajo peso molecular y acenocumarol. Se realizaron controles clínicos, hematológicos y con ecografía doppler. Se comprobó permeabilidad total de la vena previamente trombosada a los 3 meses en 5 casos (38\%), a los 4 meses en otro enfermo, a los 6 meses en dos enfermos (15\%), a los 8 meses en otros dos (15\%), a los 9 meses en otro caso. En dos enfermos la ultrasonografía de compresión venosa mostraba a los 9 meses datos de oclusión intermedia o completa por lo que no suspendimos el tratamiento anticoagulante. Si nos atenemos al tiempo aconsejado de duración de la anticoagulación para la trombosis venosa profunda proximal idiopática, que actualmente es de 6 meses, observamos que en cinco pacientes (38\%), no existían datos ecográficos de permeabilidad completa de la vena, por lo que estimamos que podría valorarse la realización de una ecografía de compresión venosa como prueba de seguimiento para comprobar la permeabilidad completa antes de suspender la anticoagulación.

\section{F. Marcos Sánchez, M. I. Albo Castaño, A. Viana Alonso, A. I. Muñoz Ruiz, S. Casallo Blanco}

Servicio de Medicina Interna. Hospital $N^{a} S^{a}$ del Prado. Talavera de la Reina, Toledo 
1. Ruiz-Giménez N, Suárez C. Diagnóstico de la enfermedad tromboembólica venosa. Emergencias 2002; 14: S42-S47.

2. Kearon C, Ginsberg JS, Hirsh J, The role of Venous Ultrasonography in the diagnosis of suspected deep venous thrombosis and pulmonary embolism. Ann Intern Med 1998; 129: 1044-9.

3. Janssen MC, Wollersheim H. Rapid D-dimer assays to exclude deep venous thrombosis and pulmonary embolism: current status and new developments. Semin Thromb Hemost 1998; 24: 393-400.

4. Siragusa S, Terulla V, Pirrelli S, Porta C, Falaschi F. A rapid D-Dimer assay in patients presenting at an emergency room with suspected acule venous trombosis: accuracy and relation to clinical variables. Haematologica $2001 ; 86: 856-61$.

5. Koopman MMW, Prandoni P, Piovella F, Ockeiford PA, Brandjes DPM, Van der Meer J, et al. Treatment of venous thrombosis with intravenous un ractionated heparin administered in the hospital as compared with subcutaneous low-molecular- weight heparin administered at home. $\mathrm{N}$ Engl J Med 1996; 334: 682-7.

6. Hyers TN, Agnelli C, Hull RD, Morris TA, Samama M, Tapson V, et al. Antitrombotic therapy for venous thromboembolism disease. Chest 2001; 119 (S): 176S-193S.

\section{Medicina Interna de enlace y consulta}

\section{Sr. Director:}

El trabajo publicado recientemente en su revista acerca de las interconsultas a medicina interna (1) evidencia una labor que debe ser básica en el diseño y funcionamiento de los servicios de medicina interna debido a su probada rentabilidad y mejora de la calidad asistencial y mortalidad. Comporta una sobrecarga asistencial para unos servicios con elevada actividad, que no suele programarse ni diseñarse al no repercutir directamente ni en su estancia media ni en su lista de espera.

Por la cantidad y calidad del trabajo asistencial de los servicios de medicina interna (2), la interconsulta debería organizarse e incluirse esta labor en el control de gestión del servicio como una innovación organizativa proponiendo benchmarking (referencias o modelos para copiar) con alternativas asistenciales como la psiquiatría de enlace y consulta $(3,4)$, la ortogeriatría $(5)$ o el hospitalista $(6,7)$, actualmente establecidas, o en trámites de serlo, como subespecialidades.

Desde el año 1975, las unidades de psiquiatría de enlace y consulta funcionan orgánica y estructuralmente en nuestros hospitales de manera coordinada con otras disciplinas médicas centradas en la detección, análisis y tratamiento de la morbilidad psiquiátrica en los pacientes médicoquirúrgicos (3). Recientemente el American Board of Psiquiatry and Neurology recomienda el estatus de subespecialidad a esta actividad y la denomina como "medicina psicosomática" (4).

Las características de los pacientes ingresados en cirugía ortopédica han favorecido la colaboración directa y programada con especialistas de pacientes mayores lo que facilita la atención, mejora la calidad y favorece el desarrollo de una subespecialidad como la ortogeriatría $(1,5)$. De hecho, las interconsultas dirigidas al Servicio de Medicina Interna son mayoritariamente de personas mayores con pluripatología ingresadas en cirugía ortopédica por fractura de cadera (1).

Por fin, en el mundo anglosajón y en los últimos años del siglo pasado, surge el movimiento hospitalista promovido fundamen- talmente por internistas que desarrollan un perfil de dedicación y atención a los pacientes ingresados, manteniendo la continuidad de cuidados como especialistas de la persona enferma y en contacto con el resto de los servicios, supervisando la atención integral del paciente durante su estancia hospitalaria $(6,7)$.

En el inventario permanentemente abierto de tareas y funciones del internista así como en las denominadas alternativas asistenciales, nuevas formas de gestión o innovaciones organizativas, debe incluirse la interconsulta y su contenido de actividad asistencial. Sea o no bajo la forma de unidades o subespecialidades, la interconsulta hospitalaria debe organizarse, programarse y dedicar personal y medios de una forma gestionada, incluida y valorada en el resto de las actividades programadas en el servicio con reparto de carga asistencial y valoración de tiempos y recursos.

\section{A. del Castillo Rueda}

Servicio de Medicina Interna II. Hospital General Universitario Gregorio Marañón. Madrid

1. García Lázaro M, Lucena Merino MM, Montero Pérez-Barquero M, Sánchez Guijo P. Estudio de la actividad asistencial de Medicina Interna en relación a las interconsultas. An Med Interna (Madrid) 2003; 20: 510-4.

2. Cardellach F. Fundamentos para la justificación de la presencia de los Servicios de Medicina Interna General en los hospitales de 3er nivel. Todo Hospital 2003; 196: 257-68.

3. Martínez Calvo A, Carrera S, Stein B, Huyse F, Herzog T, Lobo A et al. El proyecto multinacional europeo y multicéntrico español de mejora de calidad asistencial en psiquiatría de enlace en el hospital general: el perfil clínico en España. Med Clin (Barc) 2000; 115: 690-4.

4. Kornfeld DS. Consultation-liaison psychiatry: contributions to medical practice. Am J Psychiatry 2002; 159: 1964-72.

5. Amatuzzi MM, De Rosa Carelli C, Leme LE, Suzuki I. Interdisciplinary care in orthogeriatrics: a good cost-benefit model of care. J Am Geriatr Soc 2003; 51: 134-6.

6. The who, what, when, where, whom, and how of hospitalist care (Editorial). Ann Intern Med 2002; 137: 930-1.

7. Whitney JP. Hospitalist care. Ann Intern Med 2003; 139: 702.

\section{Sr. Director:}

Sirva esta carta como acuse de recibo a la publicada en la revista, con el título Medicina Interna de Enlace y Consultta, firmada por D. Alejandro del Castillo Rueda.

Queremos en primer lugar expresarle al autor de la carta nuestro agradecimiento por la buena acogida que ha mostrado a nuestra anterior publicación a la que hace referencia; y en segundo lugar y para los Servicios de Medicina Interna, apoyar su propuesta de que la interconsulta hospitalaria y su contenido de actividad asistencial "debe organizarse, programarse y dedicar personal y medios de una forma gestionada, incluida y valorada en el resto de las actividades programadas en el servicio con reparto de carga asistencial y valoración de tiempos y recursos".

\section{García Lázaro y cols.}

Servicio de Medicina Interna I. H.U.R.S. 